\title{
Remarks on conformal anti-invariant Riemannian maps to cosymplectic manifolds
}

\author{
Yllmaz Gündüzalp ${ }^{1}$ (D), Mehmet Akif Akyol $^{* 2}$ (D) \\ ${ }^{1}$ Dicle University, Department of Mathematics, 21280, Diyarbakir, Turkey \\ ${ }^{2}$ Bingol University, Faculty of Arts and Sciences, Department of Mathematics, 12000, Bingöl, Turkey
}

\begin{abstract}
M.A. Akyol and B. Şahin [Conformal anti-invariant Riemannian maps to Kaehler manifolds, U.P.B. Sci. Bull., Series A, Vol. 80, Iss. 4, 2018] defined and studied the notion of conformal anti-invariant Riemannian maps to Kaehler manifolds. In this paper, as a generalization of totally real submanifolds and anti-invariant Riemannian maps, we extend this notion to almost contact metric manifolds. In this manner, we introduce conformal anti-invariant Riemannian maps from Riemannian manifolds to cosymplectic manifolds. In order to guarantee the existence of this notion, we give a non-trivial example, investigate the geometry of foliations which are arisen from the definition of a conformal Riemannian map and obtain decomposition theorems by using the existence of conformal Riemannian maps. Moreover, we investigate the harmonicity of such maps and find necessary and sufficient conditions for conformal anti-invariant Riemannian maps to be totally geodesic. Finally, we study weakly umbilical conformal Riemannian maps and obtain a classification theorem for conformal anti-invariant Riemannian maps.
\end{abstract}

Mathematics Subject Classification (2020). 53C15, 53B20, 53D15

Keywords. cosymplectic manifold, anti-invariant Riemannian map, conformal anti-invariant Riemannian map

\section{Introduction}

In 1992, A.E. Fischer introduced Riemannian maps between Riemannian manifolds in [8] as a generalization of the notions of isometric immersions and Riemannian submersions. Let $\psi:\left(N_{1}, g_{N_{1}}\right) \rightarrow\left(N_{2}, g_{N_{2}}\right)$ be a smooth map between Riemannian manifolds such that $0<\operatorname{rank} \psi<\min \left\{n_{1}, n_{2}\right\}$, where $\operatorname{dim} N_{1}=n_{1}$ and $\operatorname{dim} N_{2}=n_{2}$. Then we denote the kernel space of $\psi_{*}$ by $k e r \psi_{*}$ and consider the orthogonal complementary space $\mathcal{H}=$ $\left(k e r \psi_{*}\right)^{\perp}$ to $k e r \psi_{*}$ in $T N_{1}$. Then the tangent bundle of $N_{1}$ has the following decomposition $T N_{1}=k e r \psi_{*} \oplus \mathcal{H}$. We denote the range of $\psi_{*}$ by range $\psi_{*}$ and consider the orthogonal complementary space $\left(\text { range }_{*}\right)^{\perp}$ to range $\psi_{*}$ in the tangent bundle $T N_{2}$ of $N_{2}$. Since $\operatorname{rank} \psi<\min \left\{n_{1}, n_{2}\right\}$, we always have $\left(\operatorname{range}_{*}\right)^{\perp}$. Thus the tangent bundle $T N_{2}$ of $N_{2}$ has the following decomposition $\psi^{-1}\left(T N_{2}\right)=$ range $_{*} \oplus\left(\text { range } \psi_{*}\right)^{\perp}$. Now, a smooth map $\psi:\left(N_{1}^{n_{1}}, g_{N_{1}}\right) \rightarrow\left(N_{2}^{n_{2}}, g_{N_{2}}\right)$ is called Riemannian map at $q_{1} \in N_{1}$ if the horizontal

\footnotetext{
*Corresponding Author.

Email addresses: ygunduzalp@dicle.edu.tr (Y. Gündüzalp), mehmetakifakyol@bingol.edu.tr (M.A. Akyol)

Received: 21.01.2020; Accepted: 12.03.2021
} 
restriction $\psi_{* q_{1}}^{h}:\left(\operatorname{ker} \psi_{* q_{1}}\right)^{\perp} \rightarrow\left(\right.$ range $\left._{* q_{1}}\right)$ is a linear isometry between the inner product spaces

$$
\left(\left(k e r \psi_{* q_{1}}\right)^{\perp},\left.g_{N_{1}}\left(q_{1}\right)\right|_{\left(k e r \psi_{* q_{1}}\right.}\right)^{\perp}
$$

and

$$
\left(\operatorname{range}_{* q_{1}},\left.g_{N_{2}}\left(q_{2}\right)\right|_{\left(\text {range }_{* q_{1}}\right)}\right), q_{2}=\psi\left(q_{1}\right) .
$$

Therefore, A. E. Fischer stated in [8] that a Riemannian map is a map which is as isometric as it can be. In another words, $\psi_{*}$ satisfies the equation

$$
g_{N_{2}}\left(\psi_{*} X_{1}, \psi_{*} X_{2}\right)=g_{N_{1}}\left(X_{1}, X_{2}\right)
$$

for $X_{1}, X_{2}$ vector fields tangent to $\mathcal{H}$. It follows that isometric immersions and Riemannian submersions are particular Riemannian maps with $\operatorname{ker} \psi_{*}=\{0\}$ and $\left(\text { range }_{*}\right)^{\perp}=\{0\}$. It is known that a Riemannian map is a subimmersion [5] and this fact implies that the rank of the linear map $\psi_{* q}: T_{q} N_{1} \rightarrow T_{\psi(q)} N_{2}$ is constant for $q$ in each connected component of $N_{1}$, [1] and [8]. It is also important to note that Riemannian maps satisfy the eikonal equation which is a bridge between geometric optics and physical optics. Different properties of Riemannian maps have been studied widely by many authors, see: $[4,9,12,14,16]$. Recent developments in the theory of Riemannian map can be found in the book [17]. Recently, conformal Riemannian maps as a generalization of Riemannian maps have been defined in [15] (see also [18]) and the harmonicity of such maps have been also obtained. One can see that conformal Riemannian maps with $k e r \psi_{*}=\{0\}$ (respectively, $\left.\left(\text { range }_{*}\right)^{\perp}=\{0\}\right)$ are conformal holomorphic submanifolds (respectively, conformal submersions). For conformal anti invariant Riemannian submersions see also $([2,13])$. The second author of the paper and B. Şahin have been defined the notion of conformal anti invariant Riemannian maps and conformal slant Riemannian maps in [3] and [4], respectively. In this paper, we are going to introduce and study the notion of conformal anti-invariant Riemannian maps from Riemannian manifolds to almost contact metric manifolds as a generalization of totally real submanifolds and anti-invariant Riemannian maps.

The paper is organized as follows. Section 2 includes preliminaries. Section 3 contains the definition of conformal Riemannian map, a proper example, the geometry of foliations determined by vertical and horizontal distributions and the geometry of leaves of these distributions.

\section{Preliminaries}

Let $N$ be an almost contact metric manifold with structure tensors $\left(\varphi, \xi, \eta, g_{N}\right)$ where $\varphi$ is a tensor field of type $(1,1), \xi$ is a vector field, $\eta$ is a 1 -form and $g_{N}$ is the Riemannian metric on $N$. Then these tensors satisfy [7]

$$
\begin{gathered}
\varphi \xi=0, \quad \eta \circ \varphi=0, \quad \eta(\xi)=1 \\
\varphi^{2}=-I+\eta \otimes \xi, \quad g_{N}\left(\varphi X_{1}, \varphi X_{2}\right)=g_{N}\left(X_{1}, X_{2}\right)-\eta\left(X_{1}\right) \eta\left(X_{2}\right),
\end{gathered}
$$

where $I$ denotes the identity endomorphism of $T N$ and $X_{1}, X_{2}$ are any vector fields on $N$. The fundamental 2 -form $\Phi$ is defined $\Phi\left(X_{1}, X_{2}\right)=g_{N}\left(X_{1}, \varphi X_{2}\right)$.

An almost contact metric structure $\left(\varphi, \xi, \eta, g_{N}\right)$ is said to be cosymplectic, if $\nabla \eta=0$ and $\nabla \Phi=0$ are closed $([7,10])$, and the structure equation of a cosymplectic manifold is given by

$$
\left(\nabla_{X_{1}} \varphi\right) X_{2}=0, \quad X_{1}, X_{2} \in \chi(N)
$$

where $\nabla$ denotes the Riemannian connection of the metric $g_{N}$ on $N$. Moreover, for a cosymplectic manifold, we know that [6]

$$
\nabla_{X_{1}} \xi=0 .
$$

We also recall the notion of harmonic maps between Riemannian manifolds. Let $\left(N_{1}, g_{N_{1}}\right)$ and $\left(N_{2}, g_{N_{2}}\right)$ be Riemannian manifolds and $\psi:\left(N_{1}, g_{N_{1}}\right) \rightarrow\left(N_{2}, g_{N_{2}}\right)$ is a 
differentiable map. Then the differential $\psi_{*}$ of $\psi$ can be viewed a section of the bundle $\operatorname{Hom}\left(T N_{1}, \psi^{-1} T N_{2}\right) \rightarrow N_{1}$, where $\psi^{-1} T N_{2}$ is the pullback bundle which has fibres $\left(\psi^{-1} T N_{2}\right)_{q}=T_{\psi(q)} N_{2}, q \in N_{1}$. Hom $\left(T N_{1}, \psi^{-1} T N_{2}\right)$ has a connection $\nabla$ induced from the Levi-Civita connection $\nabla^{N_{1}}$ and the pullback connection. The second fundamental form of $\psi$ is given by

$$
\left(\nabla \psi_{*}\right)\left(X_{1}, X_{2}\right)=\nabla_{X_{1}}^{\psi} \psi_{*} X_{2}-\psi_{*}\left(\nabla_{X_{1}}^{N_{1}} X_{2}\right)
$$

for $X_{1}, X_{2} \in \Gamma\left(N_{1}\right)$, where $\nabla^{\psi}$ is the pullback connection. It is known that the second fundamental form is symmetric. Recall that $\psi$ is said to be harmonic if $\operatorname{trace}\left(\nabla \psi_{*}\right)=0$. On the other hand, the tension field of $\psi$ is the section $\tau(\psi)$ of $\Gamma\left(\psi^{-1} T N_{2}\right)$ defined by

$$
\tau(\psi)=\operatorname{div} \psi_{*}=\sum_{i=1}^{n_{1}}\left(\nabla \psi_{*}\right)\left(e_{i}, e_{i}\right)
$$

where $\left\{e_{1}, \ldots, e_{n_{1}}\right\}$ is the orthonormal frame on $N_{1}$. Then it follows that $\psi$ is harmonic if and only if $\tau(\psi)=0[5]$.

We denote by $\nabla^{2}$ both the Levi-Civita connection of $\left(N_{2}, g_{N_{2}}\right)$ and its pullback along $\psi$. Then according to [11], for any vector field $X_{1}$ on $N_{1}$ and any section $U_{1}$ of $\left(\text { range } \psi_{*}\right)^{\perp}$, where $\left(\text { range } \psi_{*}\right)^{\perp}$ is the subbundle of $\psi^{-1} T N_{2}$ with fiber $\left(\psi_{*}\left(T_{q} N_{1}\right)\right)^{\perp}$ - orthogonal complement of $\left(\psi_{*}\left(T_{q} N_{1}\right)\right)$ for $g_{N_{2}}$ over $q$, we have $\nabla_{X_{1}}^{\psi \perp} U_{1}$ which is the orthogonal projection of $\nabla_{X_{1}}^{2} U_{1}$ on $\left(\psi_{*}\left(T_{q} N_{1}\right)\right)^{\perp}$ such that $\nabla^{\psi \perp} g_{N_{2}}=0$. We now define $\mathcal{A}_{U_{1}}$ as

$$
\nabla_{X_{1}}^{2} U_{1}=-\mathcal{A}_{U_{1}} \psi_{*} X_{1}+\nabla_{X_{1}}^{\psi \perp} U_{1}
$$

where $\mathcal{A}_{U_{1}} \psi_{*} X_{1}$ is tangential component (a vector field along $\psi$ ) of $\nabla_{X_{1}}^{2} U_{1}$. It is easy to see that $\mathcal{A}_{U_{1}} \psi_{*} X_{1}$ is bilinear in $U_{1}$ and $\psi_{*}$ and $\mathcal{A}_{U_{1}} \psi_{*} X_{1}$ at $q$ depends only on $U_{1 q}$ and $\psi_{* q} X_{1 q}$. By direct computations, we obtain $g_{N_{2}}\left(\mathcal{A}_{U_{1}} \psi_{*} X_{1}, \psi_{*} X_{2}\right)=g_{N_{2}}\left(U_{1},\left(\nabla \psi_{*}\right)\left(X_{1}, X_{2}\right)\right)$ for $X_{1}, X_{2} \in \Gamma\left(\left(k e r \psi_{*}^{\perp}\right)\right.$ and $U_{1} \in \Gamma\left(\left(\text { range } \psi_{*}\right)^{\perp}\right)$. Since $\left(\nabla \psi_{*}\right)$ is symmetric, it follows that $\mathcal{A}_{U_{1}}$ is a symmetric linear transformation of range $_{*}$.

\section{Conformal anti-invariant Riemannian maps}

We first recall that, in [15], B. Shahin shows that the second fundamental form $\left(\nabla \psi_{*}\right)\left(X_{1}, X_{2}\right), \forall X_{1}, X_{2} \in \Gamma\left(\left(\operatorname{ker} \psi_{*}\right)^{\perp}\right)$, of a conformal Riemannian map is in the following form

$$
\begin{aligned}
\left(\nabla \psi_{*}\right)\left(X_{1}, X_{2}\right)^{\text {range }_{*}} & =X_{1}(\ln \lambda) \psi_{*} X_{2}+X_{2}(\ln \lambda) \psi_{*} X_{1} \\
& -g_{N_{1}}\left(X_{1}, X_{2}\right) \psi_{*}(\operatorname{grad} \ln \lambda) .
\end{aligned}
$$

Thus if we denote the $\left(\text { range }_{*}\right)^{\perp}$-component of $\left(\nabla \psi_{*}\right)\left(X_{1}, X_{2}\right)$ by

$\left(\nabla \psi_{*}\right)\left(X_{1}, X_{2}\right)^{\text {range }_{*}}$, we can write $\left(\nabla \psi_{*}\right)\left(X_{1}, X_{2}\right)$ as

$$
\left(\nabla \psi_{*}\right)\left(X_{1}, X_{2}\right)=\left(\nabla \psi_{*}\right)\left(X_{1}, X_{2}\right)^{\text {range }_{*}}+\left(\nabla \psi_{*}\right)\left(X_{1}, X_{2}\right)^{\left(\text {range }_{*}\right)^{\perp}}
$$

for $X_{1}, X_{2} \in \Gamma\left(\left(k e r \psi_{*}\right)^{\perp}\right)$. Hence we have

$$
\begin{aligned}
\left(\nabla \psi_{*}\right)\left(X_{1}, X_{2}\right) & =X_{1}(\ln \lambda) \psi_{*} X_{2}+X_{2}(\ln \lambda) \psi_{*} X_{1}-g_{N_{1}}\left(X_{1}, X_{2}\right) \psi_{*}(\operatorname{grad} \ln \lambda) \\
& +\left(\nabla \psi_{*}\right)\left(X_{1}, X_{2}\right)^{\left(\text {range }_{*}\right)^{\perp}} .
\end{aligned}
$$

We now present the following definition for conformal anti-invariant Riemannian maps as a generalization of totally real submanifolds and anti-invariant Riemannian maps.

Definition 3.1. Let $\psi$ be a conformal Riemannian map from a Riemannian manifold $\left(N_{1}, g_{N_{1}}\right)$ to an almost contact metric manifold $\left(N_{2}, \varphi, \xi, \eta, g_{N_{2}}\right)$. Then we say that $\psi$ is a conformal anti-invariant Riemannian map at $q \in N_{1}$ if $\varphi\left(\text { range }_{*}\right)_{q} \subseteq\left(\text { range } \psi_{* q}\right)^{\perp}$. If $\psi$ is a conformal anti-invariant Riemannian map for $q \in N_{1}$, then $\psi$ is called a conformal anti-invariant Riemannian map. 
Now, we are going to give some examples of conformal anti-invariant Riemannian maps.

Example 3.2. Every anti-invariant submanifold [19] of an almost contact metric manifold is a conformal anti-invariant Riemannian map with $\lambda=1$ and $\operatorname{ker} \psi_{*}=\{0\}$.

Example 3.3. Every anti-invariant Riemannian map [16] from a Riemannian manifold to an almost contact metric manifold is a conformal anti-invariant Riemannian map with $\lambda=1$.

We say that a conformal anti-invariant Riemannian map is proper if $\lambda \neq 1$. We now present an example of a proper conformal anti-invariant Riemannian map.

Note that given an Euclidean space $N_{2}=\mathbb{R}^{5}$ with coordinates $\left(v_{1}, \ldots, v_{5}\right)$ on $N_{2}=\mathbb{R}^{5}$, we can naturally choose an almost contact structure $(\varphi, \xi, \eta)$ on $\mathbb{R}^{5}$ as follows:

$$
\begin{gathered}
\eta=d v_{5}, \quad \xi=\frac{\partial}{\partial v_{5}}, \varphi\left(\frac{\partial}{\partial v_{1}}\right)=\frac{\partial}{\partial v_{2}}, \varphi\left(\frac{\partial}{\partial v_{3}}\right)=\frac{\partial}{\partial v_{4}}, \\
\varphi\left(\frac{\partial}{\partial v_{2}}\right)=-\frac{\partial}{\partial v_{1}}, \varphi\left(\frac{\partial}{\partial v_{4}}\right)=-\frac{\partial}{\partial v_{3}}, \varphi(\xi)=0 .
\end{gathered}
$$

Example 3.4. Consider the following map defined by

$$
\psi: \mathbb{R}^{5} \rightarrow N_{2}=\mathbb{R}^{5}, \quad \psi\left(u_{1}, \ldots, u_{5}\right)=\left(e^{u_{1}} \sin u_{2}, 0, e^{u_{1}} \cos u_{2}, 0,0\right) .
$$

We have

$$
k e r \psi_{*}=\operatorname{span}\left\{U_{1}=\frac{\partial}{\partial u_{3}}, U_{2}=\frac{\partial}{\partial u_{4}}, U_{3}=\frac{\partial}{\partial u_{5}}\right\}
$$

and

$$
\begin{aligned}
\left(k e r \psi_{*}\right)^{\perp}=\operatorname{span}\left\{X_{1}\right. & =e^{u_{1}} \sin u_{2} \frac{\partial}{\partial u_{1}}+e^{u_{1}} \cos u_{2} \frac{\partial}{\partial u_{2}}, \\
X_{2} & \left.=e^{u_{1}} \cos u_{2} \frac{\partial}{\partial u_{1}}-e^{u_{1}} \sin u_{2} \frac{\partial}{\partial u_{2}}\right\} .
\end{aligned}
$$

By direct computations, we have range $\psi_{*}=\operatorname{span}\left\{\psi_{*} X_{1}=e^{2 u_{1}} \frac{\partial}{\partial v_{1}}, \psi_{*} X_{2}=e^{2 u_{1}} \frac{\partial}{\partial v_{3}}\right\}$ and $\left(\text { range }_{*}\right)^{\perp}=\operatorname{span}\left\{\frac{\partial}{\partial v_{2}}, \frac{\partial}{\partial v_{4}}, \xi=\frac{\partial}{\partial v_{5}}\right\}$. It is also easy to check that

$$
g_{N_{2}}\left(\psi_{*} X_{1}, \psi_{*} X_{1}\right)=e^{2 u_{1}} g_{N_{1}}\left(X_{1}, X_{1}\right), g_{N_{2}}\left(\psi_{*} X_{2}, \psi_{*} X_{2}\right)=e^{2 u_{1}} g_{N_{1}}\left(X_{2}, X_{2}\right),
$$

which show that $\psi$ is a conformal Riemannian map with $\lambda=e^{u_{1}}$. Moreover, it is easy to see that $\varphi \psi_{*} X_{1}=e^{2 u_{1}} \frac{\partial}{\partial v_{2}}$ and $\varphi \psi_{*} X_{2}=e^{2 u_{1}} \frac{\partial}{\partial v_{4}}$. As a result, $\psi$ is a conformal anti-invariant Riemannian map.

Remark 3.5. In this paper, we suppose that the Reeb vector field $\xi \in\left(\text { range }_{*}\right)^{\perp}$.

Let $\psi$ be a conformal anti-invariant Riemannian map from a Riemannian manifold $\left(N_{1}, g_{N_{1}}\right)$ to an almost contact metric manifold $\left(N_{2}, g_{N_{2}}, \varphi, \eta, \xi\right)$. First of all, from Definition 3.1, we have $\varphi\left(\right.$ range $\left._{*}\right) \cap\left(\text { range }_{*}\right)^{\perp} \neq\{0\}$. We denote the complementary orthogonal distribution to $\varphi\left(\right.$ range $\left.\psi_{*}\right)$ in $\left(\left(\text { range } \psi_{*}\right)^{\perp}\right)$ by $\mu$. Then we have

$$
\left(\text { range }_{*}\right)^{\perp}=\varphi\left(\text { range }_{*}\right) \oplus \mu .
$$

It is easy to see that $\mu$ is an invariant distribution of $\left(\text { range }_{*}\right)^{\perp}$, under the endomorphism $\varphi$. Thus, for $U \in \Gamma\left(\left(\text { range } \psi_{*}\right)^{\perp}\right)$, we have

$$
\varphi U=\mathcal{D} U+\mathcal{E} U
$$

where $\mathcal{D} U \in \Gamma\left(\right.$ range $\left._{*}\right)$ and $\mathcal{E} U \in \Gamma\left(\left(\text { range }_{*}\right)^{\perp}\right)$.

We now investigate the geometry of the leaves of $\left(\right.$ range $\left.\psi_{*}\right)$ and $\left(\text { range } \psi_{*}\right)^{\perp}$. 
Theorem 3.6. Let $\psi$ be a conformal anti-invariant Riemannian map from a Riemannian manifold $\left(N_{1}, g_{N_{1}}\right)$ to a cosymplectic manifold $\left(N_{2}, g_{N_{2}}, \varphi, \eta, \xi\right)$. Then $\left(\right.$ range $\left.\psi_{*}\right)$ defines a totally geodesic foliation on $N_{2}$ if and only if

$$
g_{N_{2}}\left(\left(\nabla \psi_{*}\right)\left(X_{1}, X_{3}\right)^{\left(\text {range }_{*}\right)}, \varphi \psi_{*} X_{2}\right)=g_{N_{2}}\left(\nabla_{X_{1}}^{\psi \perp} \varphi \psi_{*} X_{2}, \varepsilon U\right)
$$

for any $U \in \Gamma\left(\left(\text { range } \psi_{*}\right)^{\perp}\right)$ and $X_{1}, X_{2}, X_{3} \in \Gamma\left(\left(\text { ker } \psi_{*}\right)^{\perp}\right)$, such that $\psi_{*} X_{3}=\mathcal{D} U$.

Proof. For $U \in \Gamma\left(\left(\text { range }_{*}\right)^{\perp}\right)$ and $X_{1}, X_{2} \in \Gamma\left(\left(k e r \psi_{*}\right)^{\perp}\right)$, using (2.2), (2.3) and (2.4) we have

Thus (3.5) we obtain

$$
g_{N_{2}}\left(\nabla_{X_{1}}^{2} \psi_{*} X_{2}, U\right)=g_{N_{2}}\left(\nabla_{X_{1}}^{2} \varphi \psi_{*} X_{2}, \varphi U\right)
$$

$$
g_{N_{2}}\left(\nabla_{X_{1}}^{2} \psi_{*} X_{2}, U\right)=-g_{N_{2}}\left(\nabla_{X_{1}}^{2} \psi_{*} X_{3}, \varphi \psi_{*} X_{2}\right)+g_{N_{2}}\left(\nabla_{X_{1}}^{2} \varphi \psi_{*} X_{2}, \mathcal{E} U\right)
$$

where $\psi_{*} X_{3}=\mathcal{D} U$ for $X_{3} \in \Gamma\left(\left(k e r \psi_{*}\right)^{\perp}\right)$. Since the map is a conformal anti-invariant Riemannian map, using (2.5), (2.7) and (3.2) we obtain

$$
\begin{aligned}
g_{N_{2}}\left(\nabla_{X_{1}}^{2} \psi_{*} X_{2}, U\right) & =-g_{N_{2}}\left(\left(\nabla \psi_{*}\right)\left(X_{1}, X_{3}\right)^{\text {range }_{*}}+\left(\nabla \psi_{*}\right)\left(X_{1}, X_{3}\right)^{\left(\text {range }_{*}\right)^{\perp}}\right. \\
& \left.+\psi_{*}\left(\nabla_{X_{1}}^{N^{1}} X_{3}\right), \varphi \psi_{*} X_{2}\right) \\
& +g_{N_{2}}\left(-A_{\varphi \psi_{*}} X_{2} X_{1}+\nabla_{X_{1}}^{\psi \perp} \varphi \psi_{*} X_{2}, \mathcal{E} U\right)
\end{aligned}
$$

Hence, we arrive at

$$
\begin{aligned}
g_{N_{2}}\left(\nabla_{X_{1}}^{2} \psi_{*} X_{2}, U\right) & =-g_{N_{2}}\left(\left(\nabla \psi_{*}\right)\left(X_{1}, X_{3}\right)^{\left(\text {range }_{*}\right)^{\perp}}, \varphi \psi_{*} X_{2}\right) \\
& +g_{N_{2}}\left(\nabla_{X_{1}}^{\psi \perp} \varphi \psi_{*} X_{2}, \mathcal{E} U\right) .
\end{aligned}
$$

From above equation, $\left(\right.$ range $\left.\psi_{*}\right)$ defines a totally geodesic foliation on $N_{2}$ if and only if (3.6) is satisfied.

Theorem 3.7. Let $\psi$ be a conformal anti-invariant Riemannian map from a Riemannian manifold $\left(N_{1}, g_{N_{1}}\right)$ to a cosymplectic manifold $\left(N_{2}, g_{N_{2}}, \varphi, \eta, \xi\right)$. Then two of the assertions imply the other one:

(a) $\left(\text { range } \psi_{*}\right)^{\perp}$ defines a totally geodesic foliation on $N_{2}$.

(b) $\psi$ is a horizontally homothetic conformal Riemannian map.

(c) $g_{N_{2}}\left(\mathcal{D} U_{1}, A_{\varepsilon U_{1}} \psi_{*} X_{1}+\psi_{*}\left(\nabla_{X_{1}}^{N_{1}} X_{2}\right)\right)=-g_{N_{2}}\left(\mathcal{E} U_{2},\left(\nabla \psi_{*}\right)\left(X_{1}, X_{2}\right)^{\left(\text {range }_{*}\right)^{\perp}}\right.$

$$
\begin{aligned}
& \left.+\nabla_{X_{1}}^{\psi \perp} \mathcal{E} U_{1}\right)-g_{N_{2}}\left(U_{2},\left[U_{1}, \psi_{*} X_{1}\right]\right) \\
& -\eta\left(U_{2}\right) \eta\left(\nabla_{\psi_{*} X_{1}}^{2} U_{1}\right)
\end{aligned}
$$

for any $U_{1}, U_{2} \in \Gamma\left(\left(\text { range } \psi_{*}\right)^{\perp}\right)$ and $X_{1}, X_{2} \in \Gamma\left(\left(\text { ker } \psi_{*}\right)^{\perp}\right)$, such that $\psi_{*} X_{2}=\mathcal{D} U_{1}$.

Proof. For $U_{1}, U_{2} \in \Gamma\left(\left(\text { range } \psi_{*}\right)^{\perp}\right)$ and $X_{1} \in \Gamma\left(\left(\text { ker } \psi_{*}\right)^{\perp}\right)$, since $N_{2}$ is a cosymplectic manifold, using (2.2) and (2.3) we have

$$
\begin{aligned}
g_{N_{2}}\left(\nabla_{U_{1}}^{2} U_{2}, \psi_{*} X_{1}\right) & =-g_{N_{2}}\left(U_{2},\left[U_{1}, \psi_{*} X_{1}\right]\right)-\eta\left(U_{2}\right) \eta\left(\nabla_{\psi_{*} X_{1}}^{2} U_{1}\right) \\
& -g_{N_{2}}\left(\varphi U_{2}, \nabla_{\psi_{*} X_{1}}^{2} \varphi U_{1}\right) .
\end{aligned}
$$

Then using (3.5), (2.5) and (2.7) we obtain

$$
\begin{aligned}
& g_{N_{2}}\left(\nabla_{U_{1}}^{2} U_{2}, \psi_{*} X_{1}\right)=-g_{N_{2}}\left(U_{2},\left[U_{1}, \psi_{*} X_{1}\right]\right)-\eta\left(U_{2}\right) \eta\left(\nabla_{\psi_{*} X_{1}}^{2} U_{1}\right) \\
& -g_{N_{2}}\left(\mathcal{D} U_{2},\left(\nabla \psi_{*}\right)\left(X_{1}, X_{2}\right)+\psi_{*}\left(\nabla_{X_{1}}^{N_{1}} X_{2}\right)\right) \\
& -g_{N_{2}}\left(\mathcal{D} U_{2},-A_{\varepsilon U_{1}} \psi_{*} X_{1}\right. \\
& \left.+\nabla_{X_{1}}^{\psi \perp} \varepsilon U_{1}\right)-g_{N_{2}}\left(\varepsilon U_{2},\left(\nabla \psi_{*}\right)\left(X_{1}, X_{2}\right)+\psi_{*}\left(\nabla_{X_{1}}^{N_{1}} X_{2}\right)\right) \\
& -g_{N_{2}}\left(\mathcal{E} U_{2},-A_{\mathcal{E} U_{1}} \psi_{*} X_{1}+\nabla_{X_{1}}^{\psi \perp} \mathcal{E} U_{1}\right)
\end{aligned}
$$


where $\psi_{*} X_{2}=\mathcal{D} U_{1} \in \Gamma\left(\right.$ range $\left.\psi_{*}\right)$ for $X_{2} \in \Gamma\left(\left(\operatorname{ker} \psi_{*}\right)^{\perp}\right)$. Since $\psi$ is a conformal antiinvariant Riemannian map, using (3.2), we arrive at

$$
\begin{aligned}
g_{N_{2}}\left(\nabla_{U_{1}}^{2} U_{2}, \psi_{*} X_{1}\right) & =-g_{N_{2}}\left(U_{2},\left[U_{1}, \psi_{*} X_{1}\right]\right)-\eta\left(U_{2}\right) \eta\left(\nabla_{\psi_{*} X_{1}}^{2} U_{1}\right) \\
& -g_{N_{2}}\left(\mathcal{D} U_{2},\left(\nabla \psi_{*}\right)\left(X_{1}, X_{2}\right)^{\left(\text {range }_{*}\right)}\right)-g_{N_{2}}\left(\mathcal{D} U_{2}, \psi_{*}\left(\nabla_{X_{1}}^{N_{1}} X_{2}\right)\right) \\
& +g_{N_{2}}\left(\mathcal{D} U_{2}, A_{\mathcal{E} U_{1}} \psi_{*} X_{1}\right)-g_{N_{2}}\left(\mathcal{E} U_{2},\left(\nabla \psi_{*}\right)\left(X_{1}, X_{2}\right)^{\left(\text {range }_{*}\right)^{\perp}}\right) \\
& -g_{N_{2}}\left(\mathcal{E} U_{2}, \nabla_{X_{1}}^{\psi \perp} \mathcal{E} U_{1}\right) .
\end{aligned}
$$

Then from (3.3), we get

$$
\begin{aligned}
& g_{N_{2}}\left(\nabla_{U_{1}}^{2} U_{2}, \psi_{*} X_{1}\right)=-g_{N_{2}}\left(U_{2},\left[U_{1}, \psi_{*} X_{1}\right]\right)-\eta\left(U_{2}\right) \eta\left(\nabla_{\psi_{*} X_{1}}^{2} U_{1}\right) \\
& -g_{N_{2}}\left(\mathcal{D} U_{2}, \psi_{*}\left(\nabla_{X_{1}}^{N_{1}} X_{2}\right)\right)+g_{N_{2}}\left(\mathcal{D} U_{2}, A_{\mathcal{E} U_{1}} \psi_{*} X_{1}\right) \\
& -g_{N_{2}}\left(\mathcal{E} U_{2},\left(\nabla \psi_{*}\right)\left(X_{1}, X_{2}\right)^{\left(\text {range }_{*}\right)^{\perp}}\right)-g_{N_{2}}\left(\varepsilon U_{2}, \nabla_{X_{1}}^{\psi \perp} \mathcal{E} U_{1}\right) \\
& -g_{N_{2}}\left(\mathcal{D} U_{2}, X_{1}(\ln \lambda) \psi_{*} X_{2}+X_{2}(\ln \lambda) \psi_{*} X_{1}\right. \\
& \left.-g_{N_{1}}\left(X_{1}, X_{2}\right) \psi_{*}(\operatorname{grad} \ln \lambda)\right)
\end{aligned}
$$

or

$$
\begin{aligned}
g_{N_{2}}\left(\nabla_{U_{1}}^{2} U_{2}, \psi_{*} X_{1}\right) & =-g_{N_{2}}\left(U_{2},\left[U_{1}, \psi_{*} X_{1}\right]\right)-\eta\left(U_{2}\right) \eta\left(\nabla_{\psi_{*} X_{1}}^{2} U_{1}\right) \\
& -g_{N_{2}}\left(\mathcal{D} U_{2}, \psi_{*}\left(\nabla_{X_{1}}^{N_{1}} X_{2}\right)\right)+g_{N_{2}}\left(\mathcal{D} U_{2}, A_{\mathcal{E} U_{1}} \psi_{*} X_{1}\right) \\
& -g_{N_{2}}\left(\mathcal{E} U_{2},\left(\nabla \psi_{*}\right)\left(X_{1}, X_{2}\right)^{\left(\operatorname{range\psi }_{*}\right)^{\perp}}\right)-g_{N_{2}}\left(\mathcal{E} U_{2}, \nabla_{X_{1}}^{\psi \perp} \mathcal{E} U_{1}\right) \\
& -g_{N_{1}}\left(X_{1}, \operatorname{grad} \ln \lambda\right) g_{N_{2}}\left(\mathcal{D} U_{2}, \psi_{*} X_{2}\right) \\
& -g_{N_{1}}\left(X_{2}, \operatorname{grad} \ln \lambda\right) g_{N_{2}}\left(\mathcal{D} U_{2}, \psi_{*} X_{1}\right) \\
& +g_{N_{1}}\left(X_{1}, X_{2}\right) g_{N_{2}}\left(\mathcal{D} U_{2}, \psi_{*}(\operatorname{grad} \ln \lambda)\right) .
\end{aligned}
$$

From above equation, we can conclude that the two assertions in Theorem 3.7 imply the third.

In the sequel we are going to investigate the harmonicity of conformal anti-invariant Riemannian map.

Theorem 3.8. Let $\psi$ be a conformal anti-invariant Riemannian map from a Riemannian manifold $\left(N_{1}, g_{N_{1}}\right)$ to a cosymplectic manifold $\left(N_{2}, g_{N_{2}}, \varphi, \eta, \xi\right)$. Then $\psi$ is harmonic if the following conditions are satisfied;

(a) the fibres are minimal,

(b) $\operatorname{trace} \mathcal{D} \nabla_{(.)}^{\psi \perp} \varphi \psi_{*}()+.\psi_{*}\left(\nabla_{(.)}^{N_{1}}().\right)=0$,

(c) $\operatorname{trace} \varphi A_{\varphi \psi_{*}(.)} \psi_{*}()-.\mathcal{E} \nabla_{(.)}^{\psi \perp} \varphi \psi_{*}()=$.0 .

Proof. For $V \in \Gamma\left(k e r \psi_{*}\right)$, using (2.5), we have

$$
\left(\nabla \psi_{*}\right)(V, V)=-\psi_{*}\left(\nabla_{V}^{N_{1}} V\right) \text {. }
$$

For $Y \in \Gamma\left(\left(k e r \psi_{*}\right)^{\perp}\right)$, using (2.2), (2.3), (2.4) and (2.5), we have

$$
\left(\nabla \psi_{*}\right)(Y, Y)=\nabla_{Y}^{2} \psi_{*} Y-\psi_{*}\left(\nabla_{Y}^{N_{1}} Y\right)=-\varphi \nabla_{Y}^{2} \varphi \psi_{*} Y-\psi_{*}\left(\nabla_{Y}^{N_{1}} Y\right) .
$$

From (2.7), (3.2) and (3.5) we obtain

$$
\begin{aligned}
& \left(\nabla \psi_{*}\right)(Y, Y)^{\left(\text {range }_{*}\right)}+\left(\nabla \psi_{*}\right)(Y, Y)^{\left(\text {range }_{*}\right)^{\perp}}= \\
& \varphi \mathcal{A}_{\varphi \psi_{*} Y} \psi_{*} Y-\psi_{*}\left(\nabla_{Y}^{N_{1}} Y\right)-\mathcal{D} \nabla_{Y}^{\psi \perp} \varphi \psi_{*} Y-\mathcal{E} \nabla_{Y}^{\psi \perp} \varphi \psi_{*} Y .
\end{aligned}
$$

Then taking the $\left(\right.$ range $\left._{*}\right)$-components and $\left(\left(\text { range }_{*}\right)^{\perp}\right)-$ components of above expression (3.8), we arrive at

$$
\left(\nabla \psi_{*}\right)(Y, Y)^{\left(\text {range } \psi_{*}\right)}=-\mathcal{D} \nabla_{Y}^{\psi \perp} \varphi \psi_{*} Y-\psi_{*}\left(\nabla_{Y}^{N_{1}} Y\right) .
$$


and

$$
\left(\nabla \psi_{*}\right)(Y, Y)^{\left(\text {range }_{*}\right)^{\perp}}=-\mathcal{E} \nabla_{Y}^{\psi \perp} \varphi \psi_{*} Y+\varphi \mathcal{A}_{\varphi \psi_{*} Y} \psi_{*} Y .
$$

Then proof follows from (3.7), (3.9) and (3.10).

Now, we give necessary and sufficient conditions for a conformal anti-invariant Riemannian map to be total geodesic.

Theorem 3.9. Let $\psi$ be a conformal anti-invariant Riemannian map from a Riemannian manifold $\left(N_{1}, g_{N_{1}}\right)$ to a cosymplectic manifold $\left(N_{2}, g_{N_{2}}, \varphi, \eta, \xi\right)$. Then $\psi$ is totally geodesic if and only if

(a) $\left.g_{N_{2}}\left(\mathcal{D} \nabla_{X_{1}}^{\psi \perp} \varphi \psi_{*} X_{4}, \psi_{*} X_{5}\right)\right)=-\lambda^{2} g_{N_{1}}\left(\nabla_{X_{1}}^{N_{1}} X_{2}, X_{5}\right)$

(b) $\varphi \mathcal{A}_{\varphi \psi_{*} X_{4}} X_{1}=\mathcal{E} \nabla_{X_{1}}^{\psi \perp} \varphi \psi_{*} X$

for any $X_{1}, X_{2}=X_{3}+X_{4}, X_{5} \in \Gamma\left(T N_{1}\right)$, where $X_{4} \in \Gamma\left(\left(k e r \psi_{*}\right)^{\perp}\right), X_{3} \in \Gamma\left(k e r \psi_{*}\right)$.

Proof. For $X_{1}, X_{2} \in \Gamma\left(T N_{1}\right)$ and $X_{4} \in \Gamma\left(\left(k e r \psi_{*}\right)^{\perp}\right), X_{3} \in \Gamma\left(k e r \psi_{*}\right)$, using (2.3), (2.4), (2.5) and (2.7) we have $\left(\nabla \psi_{*}\right)\left(X_{1}, X_{2}\right)=-\varphi\left(-A_{\varphi \psi_{*} X_{4}} \psi_{*} X_{1}+\nabla_{X_{1}}^{\psi \perp} \varphi \psi_{*} X_{4}\right)-\psi_{*}\left(\nabla_{X_{1}}^{N_{1}} X_{2}\right)$. Then from (3.5) we get $\left(\nabla \psi_{*}\right)\left(X_{1}, X_{2}\right)=\varphi \mathcal{A}_{\varphi \psi_{*} X_{4}} \psi_{*} X_{1}-\mathcal{D} \nabla_{X_{1}}^{\psi \perp} \varphi \psi_{*} X_{4}-\mathcal{E} \nabla_{X_{1}}^{\psi \perp} \varphi \psi_{*} X_{4}-$ $\psi_{*}\left(\nabla_{X_{1}}^{N_{1}} X_{2}\right)$. Since $\psi$ is conformal anti-invariant Riemannian map, using (3.2), we get

$$
\begin{aligned}
& \left(\nabla \psi_{*}\right)\left(X_{1}, X_{2}\right)^{\left(\text {range }_{*}\right)}+\left(\nabla \psi_{*}\right)\left(X_{1}, X_{2}\right)^{\left(\text {range }_{*}\right)^{\perp}}= \\
& \varphi \mathcal{A}_{\varphi \psi_{*} X_{4}} \psi_{*} X_{1}-\mathcal{D} \nabla_{X_{1}}^{\psi \perp} \varphi \psi_{*} X_{4}-\mathcal{E} \nabla_{X_{1}}^{\psi \perp} \varphi \psi_{*} X_{4}-\psi_{*}\left(\nabla_{X_{1}}^{N_{1}} X_{2}\right) .
\end{aligned}
$$

Then taking the $\left(\right.$ range $\left._{*}\right)$ and $\left(\text { range } \psi_{*}\right)^{\perp}$ components we arrive at

$$
\left(\nabla \psi_{*}\right)\left(X_{1}, X_{2}\right)^{\left(\text {range }_{*}\right)}=-\mathcal{D} \nabla_{X_{1}}^{\psi \perp} \varphi \psi_{*} X_{4}-\psi_{*}\left(\nabla_{X_{1}}^{N_{1}} X_{2}\right)
$$

and

$$
\left(\nabla \psi_{*}\right)\left(X_{1}, X_{2}\right)^{\left(\text {range }_{*}\right)^{\perp}}=-\mathcal{E} \nabla_{X_{1}}^{\psi \perp} \varphi \psi_{*} X_{4}+\varphi \mathcal{A}_{\varphi \psi_{*} X_{4}} \psi_{*} X_{1}
$$

Thus $\left(\nabla \psi_{*}\right)\left(X_{1}, X_{2}\right)=0$ if and only if $\left(\nabla \psi_{*}\right)\left(X_{1}, X_{2}\right)^{\left(\text {range } \psi_{*}\right)}=0$ and $\left(\nabla \psi_{*}\right)\left(X_{1}, X_{2}\right)^{\left(\text {range }_{*}\right)^{\perp}}=0$. Hence we have $\left.g_{N_{2}}\left(\mathcal{D} \nabla_{X_{1}}^{\psi \perp} \varphi \psi_{*} X_{4}, \psi_{*} X_{5}\right)\right)=$ $-\lambda^{2} g_{N_{1}}\left(\nabla_{X_{1}}^{N_{1}} X_{2}, X_{5}\right)$ and $\varphi \mathcal{A}_{\varphi \psi_{*} X_{4}} \psi_{*} X_{1}-\mathcal{E} \nabla_{X_{1}}^{\psi \perp} \varphi \psi_{*} X_{4}=0$, which complete the proof.

Also, we have the following result for totally geodesic conformal anti-invariant Riemannian maps.

Theorem 3.10. Let $\psi$ be a conformal anti-invariant Riemannian map from a Riemannian manifold $\left(N_{1}, g_{N_{1}}\right)$ to a cosymplectic manifold $\left(N_{2}, g_{N_{2}}, \varphi, \eta, \xi\right)$. Then $\psi$ is totally geodesic if and only if

(a) The horizontal distribution $\left(k e r \psi_{*}\right)^{\perp}$ defines a totally geodesic foliation on $N_{1}$.

(b) All the fibres $\psi^{-1}\left(q_{2}\right)$ are totally geodesic for $q_{2} \in N_{2}$.

(c) $g_{N_{2}}\left(\left(\nabla \psi_{*}\right)\left(X_{1}, X_{3}\right)^{\left(\text {range }_{*}\right)^{\perp}}, \varphi \psi_{*} X_{2}\right)=g_{N_{2}}\left(\left(\nabla_{X_{1}}^{\psi \perp} \varphi \psi_{*} X_{2}, \mathcal{E} U\right)\right.$

for any $X_{1}, X_{2}, X_{3} \in \Gamma\left(\left(k e r \psi_{*}\right)^{\perp}\right)$, and $U \in \Gamma\left(\text { range } \psi_{*}\right)^{\perp}$.

Proof. For $X_{1}, X_{2} \in \Gamma\left(\left(k e r \psi_{*}\right)^{\perp}\right)$, and $V \in \Gamma\left(\operatorname{ker} \psi_{*}\right)$, using (2.5), we have

$$
g_{N_{2}}\left(\left(\nabla \psi_{*}\right)\left(X_{1}, V\right), \psi_{*} X_{2}\right)=-\lambda^{2} g_{N_{1}}\left(\nabla_{X_{1}}^{N_{1}} V, X_{2}\right) .
$$

$\nabla^{N_{1}}$ is a Levi-Civita connection, we obtain

$$
g_{N_{2}}\left(\left(\nabla \psi_{*}\right)\left(X_{1}, V\right), \psi_{*} X_{2}\right)=\lambda^{2} g_{N_{1}}\left(\nabla_{X_{1}}^{N_{1}} X_{2}, V\right) .
$$


Hence $\left(\nabla \psi_{*}\right)\left(X_{1}, V\right)=0$ for $X_{1} \in \Gamma\left(\left(k e r \psi_{*}\right)^{\perp}\right)$ and $V \in \Gamma\left(k e r \psi_{*}\right)$ if and only if (a). For $Y \in \Gamma\left(\left(\operatorname{ker} \psi_{*}\right)^{\perp}\right)$ and $U_{1}, U_{2} \in \Gamma\left(\operatorname{ker} \psi_{*}\right)$, we have

$$
g_{N_{2}}\left(\left(\nabla \psi_{*}\right)\left(U_{1}, U_{2}\right), \psi_{*} Y\right)=-\lambda^{2} g_{N_{1}}\left(\nabla_{U_{1}}^{N_{1}} U_{2}, Y\right)
$$

Thus $\left(\nabla \psi_{*}\right)\left(U_{1}, U_{2}\right)=0$ for $U_{1}, U_{2} \in \Gamma\left(k e r \psi_{*}\right)$ if and only if (b).

For $X_{1}, X_{2} \in \Gamma\left(\left(k e r \psi_{*}\right)^{\perp}\right)$ and $U \in \Gamma\left(\text { range }_{*}\right)^{\perp}$, since $N_{2}$ is a cosymplectic manifold, using (2.2), (2.3), (2.5) and (3.5) we have

$$
g_{N_{2}}\left(\left(\nabla \psi_{*}\right)\left(X_{1}, X_{2}\right), U\right)=-g_{N_{2}}\left(\left(\nabla_{X_{1}}^{2} \psi_{*} X_{3}, \varphi \psi_{*} X_{2}\right)+g_{N_{2}}\left(\left(\nabla_{X_{1}}^{2} \varphi \psi_{*} X_{2}, \mathcal{E} U\right),\right.\right.
$$

where $\psi_{*} X_{3}=\mathcal{D} U$ for $X_{3} \in \Gamma\left(\left(k e r \psi_{*}\right)^{\perp}\right)$. Since $\psi$ is a conformal anti-Riemannian map, using (2.5), (2.7) and (3.2) we obtain

$$
\begin{aligned}
g_{N_{2}}\left(\left(\nabla \psi_{*}\right)\left(X_{1}, X_{2}\right), U\right) & =-g_{N_{2}}\left(\left(\nabla \psi_{*}\right)\left(X_{1}, X_{3}\right)^{\left(\text {range }_{*}\right)^{\perp}}, \varphi \psi_{*} X_{2}\right) \\
& +g_{N_{2}}\left(\left(\nabla_{X_{1}}^{\psi \perp} \varphi \psi_{*} X_{2}, \mathcal{E}\right) .\right.
\end{aligned}
$$

Thus, $\left(\nabla \psi_{*}\right)\left(X_{1}, X_{2}\right)=0$ for $X_{1}, X_{2} \in \Gamma\left(\left(k e r \psi_{*}\right)^{\perp}\right)$ if and only if (c).

Now, we investigate the umbilical case in [11] for the conformal anti-invariant Riemannian maps.

Let $\psi$ be a map from a Riemannian manifold $\left(N_{1}, g_{N_{1}}\right)$ to a Riemannian manifold $\left(N_{2}, g_{N_{2}}\right)$. Then $\psi$ is called a weakly $g_{N 1}$-umbilical if there exist

a) a field $X_{3}$ along $\psi$, nowhere, 0 , with values in range $\psi_{*}$,

b) a field $X_{4}$ on $N_{1}$ such that for every $X_{1}, X_{2}$ on $\Gamma\left(T N_{1}\right)$ we have

$$
\left(\nabla \psi_{*}\right)\left(X_{1}, X_{2}\right)=g_{N_{1}}\left(X_{1}, X_{2}\right)\left[\psi_{*} X_{4}+X_{3}\right] .
$$

$\psi$ is called strong $g_{N 1}$-umbilical if $X_{4}=0$.

Using the above definition, we can give the following theorem.

Theorem 3.11. Let $\psi$ be a weakly $g_{N_{1}}$-umbilical conformal anti-Riemannian map from a Riemannian manifold $\left(N_{1}, g_{N_{1}}\right)$ to a cosymplectic manifold $\left(N_{2}, g_{N_{2}}, \varphi, \eta, \xi\right)$ such that $\operatorname{dim}(\mathcal{H}) \geq 2$. Then $\psi$ is totally geodesic map.

Proof. We suppose that $\psi$ is a weakly $g_{N_{1}}$-umbilical conformal anti-Riemannian map such that $\operatorname{dim}(\mathcal{H}) \geq 2$. Then from (3.3) and (3.11) we have

$$
X_{1}(\ln \lambda) \psi_{*} X_{2}+X_{2}(\ln \lambda) \psi_{*} X_{1}-g_{N_{1}}\left(X_{1}, X_{2}\right) \psi_{*}(\operatorname{grad} \ln \lambda)=g_{N_{1}}\left(X_{1}, X_{2}\right) \psi_{*} X_{4}
$$

and

$$
\left(\nabla \psi_{*}\right)\left(X_{1}, X_{2}\right)^{\left(\text {range }_{*}\right)^{\perp}}=g_{N_{1}}\left(X_{1}, X_{2}\right) X_{3}
$$

for $X_{1}, X_{2} \in \Gamma\left(\left(k e r \psi_{*}\right)^{\perp}\right)$. Since $\operatorname{dim}(\mathcal{H}) \geq 2$, we can choose $X_{1}$ and $X_{2}$ such that $g_{N_{1}}\left(X_{1}, X_{2}\right)=0$. Then we get

$$
X_{1}(\ln \lambda) \psi_{*} X_{2}+X_{2}(\ln \lambda) \psi_{*} X_{1}=0 .
$$

Since $X_{1}$ and $X_{2}$ are orthogonal and $\psi$ is a conformal anti-Riemannian map, we have $g_{N_{2}}\left(\psi_{*} X_{1}, \psi_{*} X_{2}\right)=\lambda^{2} g_{N_{1}}\left(X_{1}, X_{2}\right)=0 . \psi_{*} X_{1}$ and $\psi_{*} X_{2}$ are also orthogonal. Then we get

$$
X_{1}(\ln \lambda) \psi_{*} X_{2}=0, X_{2}(\ln \lambda) \psi_{*} X_{1}=0 .
$$

Thus $\psi$ is a horizontally homothetic Riemannian map. Since $\psi$ is horizontally homothetic Riemannian map, from (3.12), we get $X_{4}=0$. Thus $\left(\nabla \psi_{*}\right)\left(X_{1}, X_{2}\right)=g_{N_{1}}\left(X_{1}, X_{2}\right) X_{3}$ for $X_{1}, X_{2} \in \Gamma\left(T N_{1}\right)$. In particular, for $U_{1}, U_{2} \in \Gamma\left(k e r \psi_{*}\right)$, we get $-\psi_{*}\left(\nabla_{U_{1}} U_{2}\right)=$ $g_{N_{1}}\left(U_{1}, U_{2}\right) X_{3}$. The right side of this equation belongs to $\Gamma\left(\left(\text { range }_{*}\right)^{\perp}\right)$ while the left side of this equation belongs to $\Gamma\left(\right.$ range $\left._{*}\right)$. Hence $\psi_{*}\left(\nabla_{U_{1}} U_{2}\right)=0$ and $X_{3}=0$ which proves our assertion.

From Theorem 3.10 and Theorem 3.11, we have: 
Corollary 3.12. Let $\psi$ be a strong $g_{N_{1}}$-umbilical conformal anti-invariant Riemannian map from a Riemannian manifold $\left(N_{1}, g_{N_{1}}\right)$ to a cosymplectic manifold $\left(N_{2}, g_{N_{2}}, \varphi, \eta, \xi\right)$ such that $\operatorname{dim}(\mathcal{H}) \geq 2$. Then we have the following:

(a) The horizontal distribution $\left(k e r \psi_{*}\right)^{\perp}$ defines a totally geodesic foliation on $N_{1}$.

(b) All the fibres $\psi^{-1}\left(q_{2}\right)$ are totally geodesic for $q_{2} \in N_{2}$.

(c) $\left(\text { range } \psi_{*}\right)^{\perp}$ defines a totally geodesic foliation on $N_{2}$.

Acknowledgment. The authors are grateful to the referees for their constructive suggestions and comments.

\section{References}

[1] R. Abraham, J.E. Marsden and T. Ratiu, Manifolds, Tensor Analysis and Applications, Appl. Math. Sci. Vol. 75, Springer, New York, 1988.

[2] M.A. Akyol, Conformal anti-invariant Riemannian submersions from cosymplectic manifolds, Hacet. J. Math. Stat. 46 (2), 177-192, 2017.

[3] M.A. Akyol and B. Șahin, Conformal anti-invariant Riemannian maps to Kahler manifolds, U.P.B. Sci. Bull., Series A. 80 (4), 187-198, 2018.

[4] M.A. Akyol and B. Sahin, Conformal slant Riemannian maps to Kahler manifolds, Tokyo J. Math. 42 (1), 225-237, 2019.

[5] P. Baird and J.C. Wood, Harmonic Morphisms Between Riemannian Manifolds, Clarendon Press, Oxford, 2003.

[6] D.E. Blair, The theory of quasi-Sasakian structure, J. Differential Geom. 1, 331-345, 1967.

[7] D.E. Blair, Contact Manifolds in Riemannian Geometry, Lectures Notes in Mathematics 509, Springer-Verlag, Berlin, 1976.

[8] A.E. Fischer, Riemannian maps between Riemannian manifolds, Contemp. Math. 132, 331-366, 1992.

[9] J.P. Jaiswal, Harmonic maps on Sasakian manifolds, J. Geom. 104 (2), 309-315, 2013.

[10] G.D. Ludden, Submanifolds of cosymplectic manifolds, J. Differential Geom. 4, 237244, 1970.

[11] T. Nore, Second fundamental form of a map, Ann. Mat. Pura Appl. 146, 281-310, 1987.

[12] B. Pandey, J.P. Jaiswal and R.H. Ojha, Necessary and Sufficient Conditions for the Riemannian Map to be a Harmonic Map on Cosymplectic Manifolds, Proc. Natl. Acad. Sci., India, Sect. A Phys. Sci. 85 (2), 265-268, 2015.

[13] K.S. Park, H-conformal anti-invariant submersions from almost quaternionic Hermitian manifolds, Czechoslovak Math. J. 70, 631-656, 2020.

[14] R. Prasad and S. Pandey, Slant Riemannian maps from an almost contact manifold, Filomat, 31 (13), 3999-4007, 2017.

[15] B. Sahin, Conformal Riemannian maps between Riemannian manifolds, their harmonicity and decomposition theorems, Acta Appl. Math. 109 (3), 829-847, 2010.

[16] B. Șahin, Invariant and anti-invariant Riemannian maps to Kahler manifolds, Int. J. Geom. Methods Mod. Phys. 7 (3), 1-19, 2010.

[17] B. Şahin, Riemannian Submersions, Riemannian Maps in Hermitian Geometry, and their Applications, Elsevier, Academic Press, 2017.

[18] B. Șahin and Ş. Yanan, Conformal Riemannian maps from almost Hermitian manifolds, Turkish J. Math. 42 (5), 2436-2451, 2018.

[19] K. Yano and M. Kon, Anti-invariant submanifolds, Lect. Notes Pure Appl. Math. Vol. 21, Marcel Dekker Inc., 1976. 